\title{
Gemeinsam sind wir stark
}

Am 1. Juni um 15.26 Uhr war es dann endlich klar: Das Schweizer Stimmvolk hat am vergangenen Wochenende dem Verfassungsartikel mit 69,5\% und damit dem Machtanspruch der Kassen eine deutliche Absage erteilt. Und die Schweizer Stimmbürgerinnen und Stimmbürger haben damit auch unmissverständlich Ja gesagt zur freien Arztwahl!

Begonnen hat die Ärzteschaft diesen Abstimmungskampf vor einigen Monaten: Die Delegiertenversammlung ist der Empfehlung des FMH-Zentralvorstands gefolgt und hat sich für eine aktive Rolle der FMH im Abstimmungskampf gegen den Verfassungsartikel ausgesprochen. Von Anfang an war klar, dass die FMH diesen Kampf nicht im Alleingang führt, sondern Koalitionen mit anderen Leistungserbringerorganisationen bildet eine grossartige Chance! So ist das Komitee «NEIN zum Kassendiktat» entstanden - und damit eine breitabgestützte und glaubwürdige Gegnerschaft gegen den Verfassungsartikel: Ärztinnen und Ärzte, Patienten- und Konsumentenschutzorganisationen, Apotheker, Zahnärzte, Pflegefachfrauen und -männer, Hebammen, Physiotherapeutinnen und -therapeuten sind gemeinsam gegen die Abstimmungsvorlage angetreten. In enger Kooperation mit den Kantonen bzw. den Gesundheitsdirektoren sowie den politischen Parteien konnte nach und nach ein Meinungsumschwung erreicht werden.

Einen zentralen und vielleicht gar den wichtigsten Beitrag in diesem Abstimmungskampf haben Sie als Ärztin bzw. Arzt geleistet! Dafür möchte ich mich im Namen der FMH und des Komitees «NEIN zum Kassendiktat» herzlich bedanken! Flyers, Plakate und Buttons sind im ärztlichen Umfeld zum Einsatz gekommen, um den Patientinnen und den Patienten die Kehrseite des Verfassungsartikels bewusst zu machen. Viele von Ihnen sind zu wahren Abstimmungskämpferinnen und -kämpfern geworden, haben Testimonialinserate geschaltet und Leserbriefe geschrieben, sind kantonalen Komitees beigetreten oder haben sich Standaktionen angeschlossen.
Darüber hinaus haben manche Ärzteorganisationen zusätzliche Aktivitäten an die Hand genommen und umgesetzt. Ein grosses Dankeschön auch an all jene Spenderinnen und Spender, die die Kampagne finanziell unterstützt haben!

Als ich die Kommunikationsleitung der FMH im August 2007 antrat, wusste ich vor allem in der Theorie, wie heterogen sich die Ärztelandschaft präsentiert. Im Verlaufe meiner Tätigkeit wurde mir zunehmend bewusst, dass Partikularinteressen und föderalistische Strukturen einen einheitlichen Auftritt eines starken Dachverbandes (die FMH zählt über 30000 Mitglieder) erschweren würden. Dazu kam, dass an der Ärztekammer vom Mai 2007 ein Projekt zur Imagebildung aus verschiedenen, auch nachvollziehbaren Gründen abgelehnt worden war.

Auch dieses Blatt hat sich gewendet, denn die Abstimmungskampagne hat mit Sicherheit einen wesentlichen Beitrag zur Imagebildung geleistet. Und zwar gegen aussen, indem die FMH, die Verbindung von Ärztinnen und Ärzten, ins Gespräch gekommen ist - sowohl auf der politischen als auch medialen Bühne und damit auch in der breiten Öffentlichkeit. Dieses kommunikative Netzwerk gilt es zu pflegen und weiter auszubauen, um unsere Ideen und Ziele an zentralen Stellen einbringen zu können. Einen wesentlichen Beitrag zur Imagebildung hat die Abstimmungskampagne auch gegen innen geleistet: Selten ist die Schweizer Ärzteschaft - Vertreter der Grundversorger und Spezialistinnen, praktizierende Ärzte und Spitalärzte, Vertreterinnen und Vertreter aller Landesteile so geeint aufgetreten. Wieviel Schlagkraft die Ärzteschaft dadurch gewonnen hat, zeigt die bereits vergangene $\mathrm{Ab}$ stimmung. Diese Schlagkraft zu behalten, um im Schweizerischen Gesundheitswesen weiterhin die Rolle eines Keyplayers einzunehmen - das muss auch in der Zukunft unser Ziel bleiben.

Jacqueline Wettstein, Leiterin Kommunikation FMH 